\title{
Characterization of a Drosophila protein associated with boundaries of transcriptionally active chromatin
}

\author{
David T. Champlin, ${ }^{1}$ Manfred Frasch, ${ }^{2}$ Harald Saumweber, ${ }^{2,3}$ and John T. Lis ${ }^{4}$ \\ ${ }^{1}$ Section of Biochemistry, Molecular and Cell Biology, Cornell University, Ithaca, New York 14853 USA; ${ }^{2}$ Max Planck \\ Institut für Entwicklungsbiologie, D-7400 Tübingen, Germany
}

\begin{abstract}
We have used indirect immunofluorescence of polytene chromosomes to examine the chromatin distribution of a 52-kD Drosophila protein designated B52. B52 is localized to transcriptionally active loci and, at the highly decondensed heat shock loci, can be seen to bracket the RNA polymerase II fluorescence signals symmetrically. We have also examined the distribution of B52 on nonpolytene chromosomes in Drosophila cell cultures with an in vivo UV cross-linking method and find that, here too, B52 is associated with boundaries of transcriptionally active chromatin. The predicted primary amino acid sequence of B52 reveals two regions with similarities to a number of other proteins known to interact with nucleic acids.
\end{abstract}

[Key Words: Drosophila; B52 protein; chromatin; heat shock]

Received March 18, 1991; revised version accepted June 20, 1991.

It is difficult to imagine how typical interphase chromatin could serve as a substrate for transcription. The DNA template is wound over the surface of nucleosomes and the nucleosomes are, in turn, packed into a coiled 30-nm fiber. RNA polymerase, which is larger than the nucleosome, not only must penetrate this higher order structure but also transcribe a helical DNA template bound to the surface of nucleosomes. On the basis of geometric considerations alone, one would predict that transcription, or the preparation for transcription, dramatically alters chromatin structure. Consistent with this notion, the transition of a gene from an inactive to an active state is accompanied by biochemically detectable changes in the organization of chromatin (for review, see van Holde 1988). Highly transcribed genes exhibit the most pronounced perturbations. Protein-DNA crosslinking experiments have shown that although histones are retained on such genes (Solomon et al. 1988), the organization of the nucleosome is altered (Nacheva et al. 1989). Consistent with this, examination of the chromatin axis by electron microscopy reveals that the DNA of highly transcribed genes is in an almost fully extended conformation (Bjorkroth et al. 1988). The opened, decondensed structure associated with transcriptionally active chromatin is detectable as a general increase in the accessibility to DNA of a variety of DNA-modifying agents including DNase I (for review, see Eisenberg et al. 1985). This accessibility extends beyond the transcribed region,

${ }^{3}$ Present address: Institut für Entwicklungsphysiologie, Universitat zu Köln, D-5000 Koln 41, Germany.

${ }^{4}$ Corresponding author. indicating that some of the structural changes associated with transcription are not a simple consequence of the passing of an RNA polymerase molecule.

Changes in the molecular composition of chromatin accompanying these structural alterations include modifications of existing components [e.g., the histones are hyperacetylated [Csordas 1990)] and the appearance of additional proteins. In the heat shock response, for example, these additional proteins include specific transcription factors like heat shock factor (Wu 1984; Thomas and Elgin 1988) and proteins that play roles in the transcription of a wide array of genes. A well-characterized example of the latter is topoisomerase I, which has been shown to be recruited to transcriptionally activated genes including the heat shock genes by indirect immunofluorescence of polytene chromosomes (Fleischmann et al. 1984) and by an in vivo UV cross-linking method (Gilmour et al. 1986). Topoisomerase I activity increases at these loci (Gilmour and Elgin 1987); furthermore, genetic analysis in yeast has shown that the topoisomerase I gene is required for high levels of transcription (Brill et al. 1987).

The identification and characterization of other proteins specifically associated with transcribed chromatin will provide a better understanding of the structural changes in chromatin that accompany gene activation. A rare opportunity to identify such proteins is provided by the polytene chromosomes of Drosophila, which possess regions of visibly decondensed chromatin, known as puffs, at many highly transcribed loci. We have screened members of a library of monoclonal antibodies (Frasch 1985) using an indirect immunofluorescence assay (Sil- 
ver and Elgin 1976) to identify new proteins associated with these puffs. One of these proteins, designated B52, is associated with transcriptionally active loci and can be seen on a number of large heat shock puffs to flank the RNA polymerase II fluorescence signals symmetrically. The bracketing of RNA polymerase II by B 52 is similar at loci containing various numbers of heat shock genes and suggests that the protein identifies the boundaries of decondensed chromatin. We have extended these observations to nonpolytene chromosomes by use of a high resolution, in vivo UV cross-linking method developed by Gilmour and Lis (Gilmour and Lis 1985; Gilmour et al. 1986). The distribution of $\mathrm{B} 52$ at the $87 \mathrm{~A}$ and 67B heat shock loci appears to be similar on both types of chromosomes and indicates that B52 is associated with boundaries of transcriptionally active chromatin. We have cloned and sequenced cDNA encoding the B52 protein and find two regions that have similarities to proteins known to interact with nucleic acids. One of these contains a good match to the consensus shared by a number of RNA- and single-stranded DNA-binding proteins. On the basis of our observations of the distribution and properties of the protein, we suggest that $\mathrm{B} 52$ may play a general role in the condensation or decondensation of chromatin.

\section{Results}

B52 is associated with transcriptionally active chromatin

The generation of a large series of monoclonal antibodies directed against nuclear antigens of Drosophila has been described previously (Frasch 1985; Frasch and Saumweber 1989). We examined polytene chromosomes by indirect immunofluorescence to identify antibodies within this series that recognize proteins associated with transcriptionally active chromatin. One antibody recognized a protein, which we call $\mathrm{B} 52$ for $52-\mathrm{kD}$ bracketing protein. B52 is broadly distributed on interbands and puffs on chromosomes from non-heat-shocked larvae, and, upon heat shock, B52 is concentrated on heat shock puffs (Fig. 1).

Figure 2 shows a comparison of the distribution of RNA polymerase II and B52 on polytene chromosomes. In unstressed animals, both proteins appear, in general,
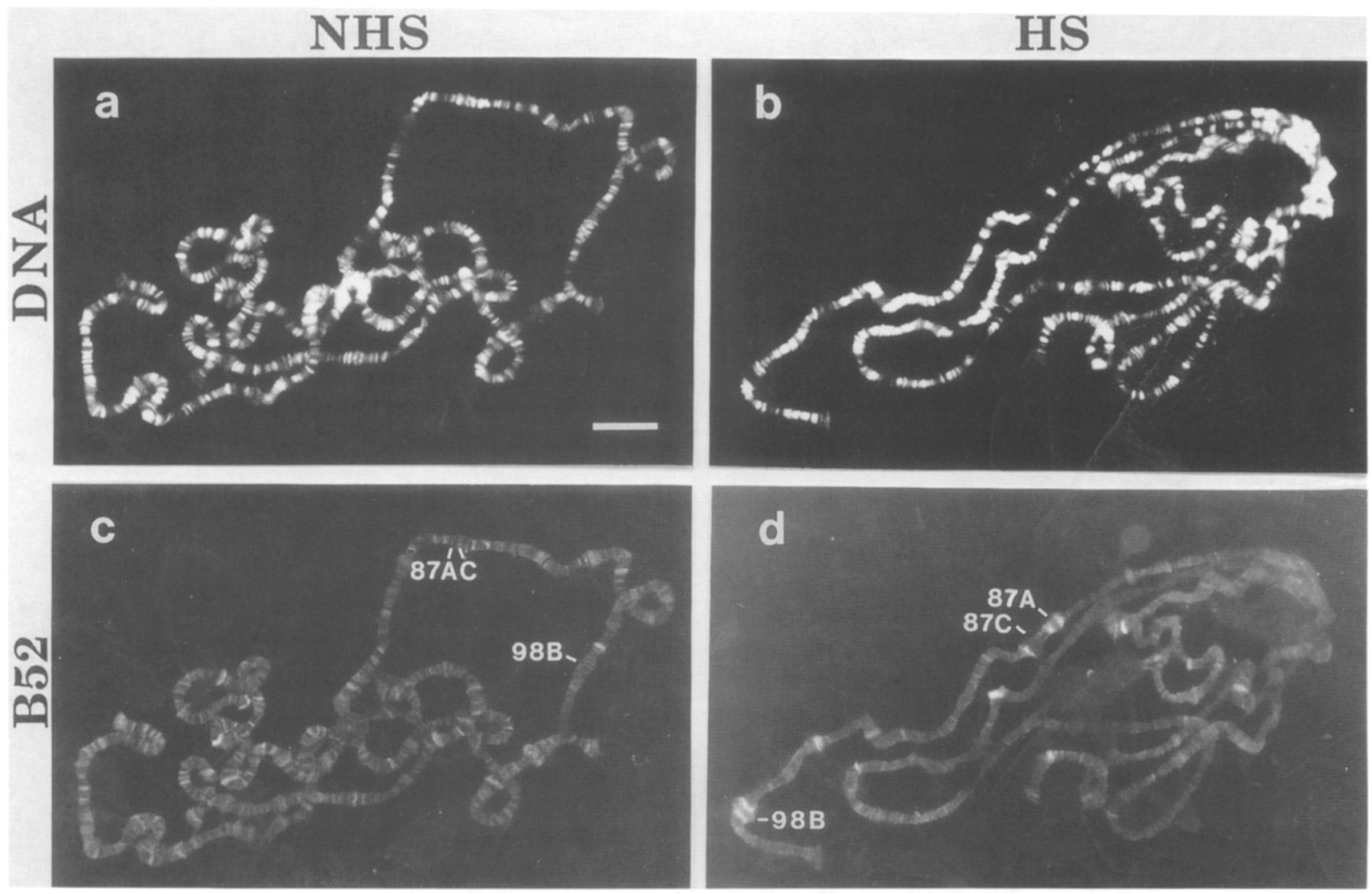

Figure 1. Distribution of B52 on polytene chromosomes. Indirect immunofluorescence of B52 on salivary gland polytene chromosomes from non-heat-shocked (NHS) or heat-shocked (HS) third-instar larvae. The same chromosomes are in each column. $(a, b)$ DNA stained with Hoechst 33258 ; $(c, d)$ B52 stained with the anti-B52 monoclonal antibody and a secondary anti-mouse antibody conjugated to rhodamine. The cytogenetic loci containing some of the heat shock genes are labeled. The chromomes are from transformant line cHB $\Delta-89$, which contains an $h s p 70-1 a c Z$ gene at the 98B locus (Simon et al. 1985a). Bar, $10 \mu \mathrm{M}$. 

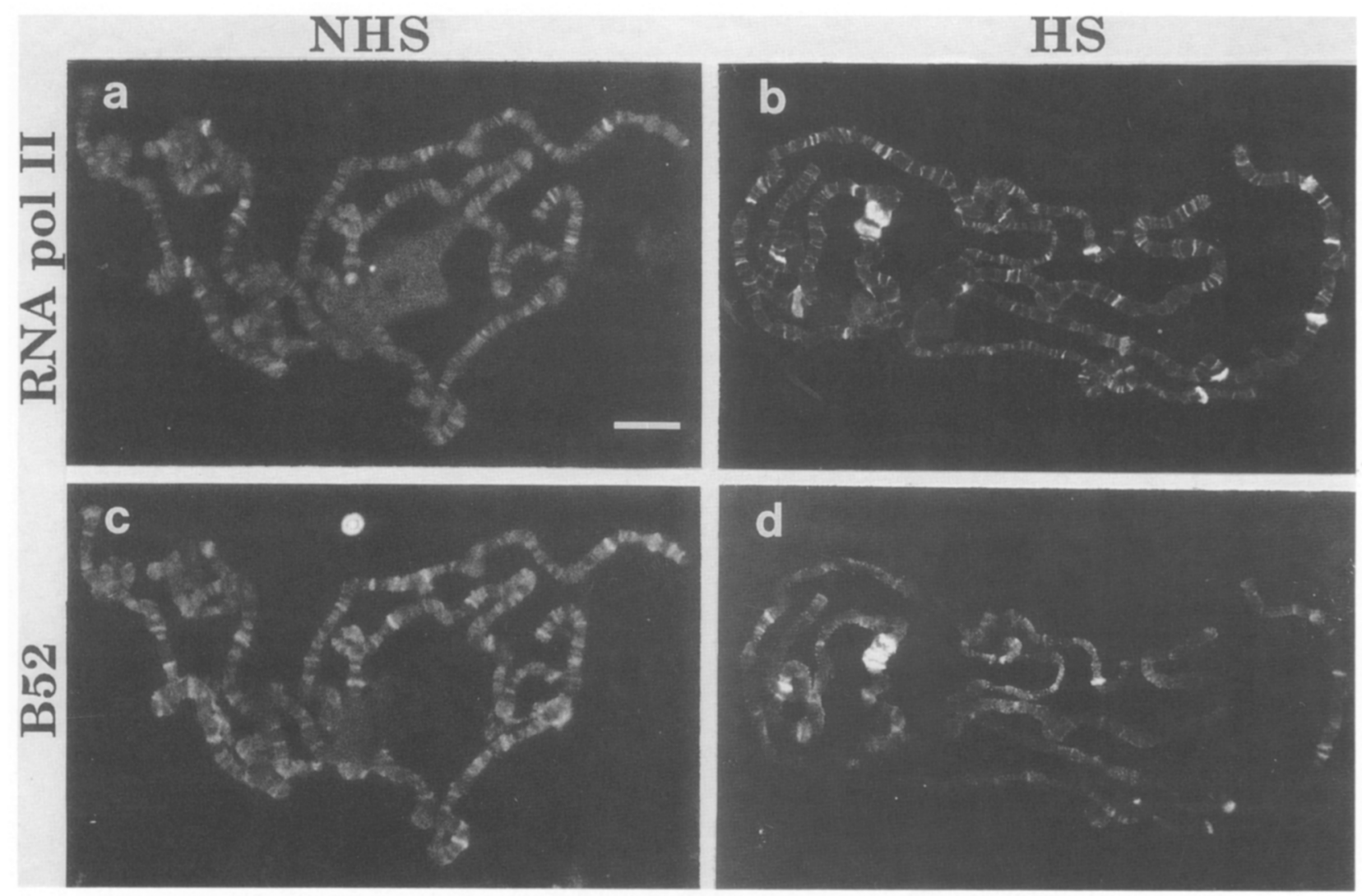

Figure 2. Distribution of B52 and RNA polymerase II on polytene chromosomes. Double-label indirect immunofluorescence of B52 and RNA polymerase II on salivary gland polytene chromosomes from non-heat-shocked (NHS) or heat-shocked (HS) third-instar larvae. The same chromosomes are in each column. $(a, b)$ RNA polymerase II stained with anti-RNA polymerase II polyclonal antibody and a secondary anti-rabbit antibody conjugated to fluorescein; $(c, d)$ B52 stained with the anti-B52 monoclonal antibody and a secondary anti-mouse antibody conjugated to rhodamine. Bar, $10 \mu \mathrm{M}$.

to be colocalized. The distribution of RNA polymerase II is profoundly altered upon heat shock (Jamrich et al. 1977), reflecting a general decrease in transcription of non-heat-shock genes and the activation of transcription of the heat shock genes. A corresponding change in the distribution of B52 is seen. At heat shock loci in particular, both proteins are present at very low levels in unstressed animals and increase dramatically in response to heat shock activation.

Although the two proteins generally appear to be colocalized (Fig. 3A), the B52 signal is broader than that of RNA polymerase II at a number of developmental and heat shock puffs; and at the largest heat shock puffs, the B52 signals bracket the RNA polymerase II signal symmetrically (Fig. 3B). Two other methods of preparing salivary gland polytene chromosomes for immunofluorescence gave similar results (Silver and Elgin 1978; Zink and Paro 1989). At loci where bracketing can be detected, the signal for B52 is immediately adjacent to that for RNA polymerase II, and the two signals may overlap. At these loci, the B52 signal appears to be equal on both sides of the RNA polymerase II signal. The only exception to this is at the $87 \mathrm{C}$ heat shock locus where the B52 signal on the proximal side of the locus is variable, perhaps because of its proximity to the $87 \mathrm{~A}$ puff.
A single set of symmetric brackets is seen at each locus even though the heat shock genes responsible for the formation of each puff vary in size and number (Fig. 3B). For example, a single heat shock gene, $h s p 68$, is present in the puff formed at the 95D locus, yet the puff is bracketed in a manner similar to the $87 \mathrm{~A}$ and $87 \mathrm{C}$ puffs, each of which contains multiple copies of the $h s p 70$ gene. The bracketing of RNA polymerase II by B52 is also seen at the new heat shock puffs formed in transformant Drosophila lines containing either the Escherichia coli lacZ gene (Simon et al. 1985a) or the yolk protein 1 gene (Kraus et al. 1988), which have been rendered heat-inducible by fusion to the $h s p 70$ promoter (Figs. 1 and $3 \mathrm{~B}$ ). Because the only sequence similarity between these transformant constructs and endogenous heat shock genes is in the promoter, this observation suggests that the distribution of B52 is not a consequence of its binding to a specific DNA sequence.

\section{B52 has a similar distribution at heat shock loci on nonpolytene chromosomes}

To examine B52 on nonpolytene chromosomes, we used the technique of in vivo protein-DNA cross-linking (Gilmour and Lis 1985; Gilmour et al. 1986) with Schneider 


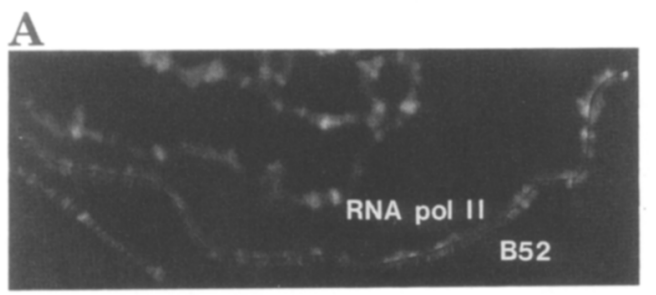

B

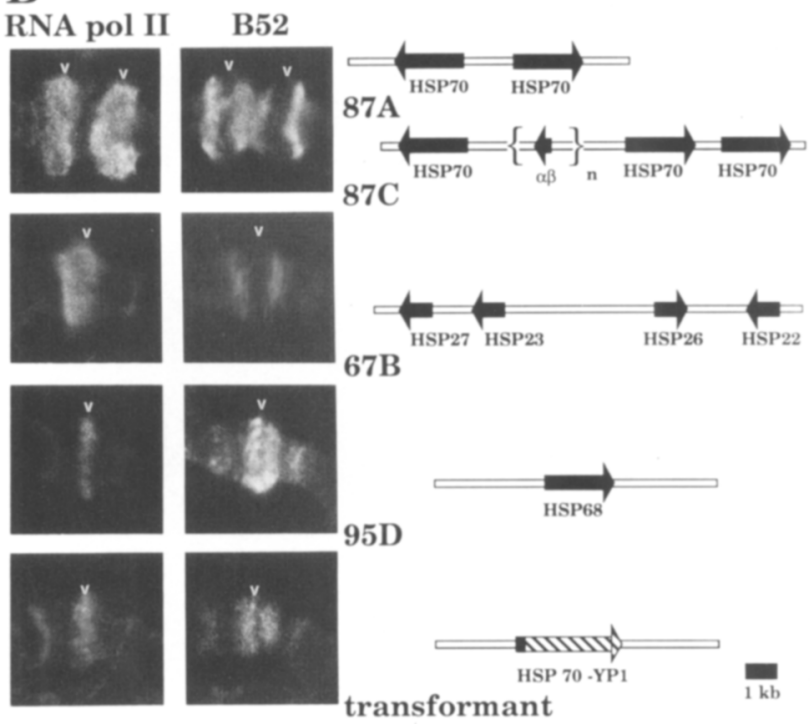

Figure 3. Comparision of RNA polymerase II and B52 at specific loci. RNA polymerase II and B52 were detected as in Fig. 2. (A) A composite fluorescent image of an $\mathrm{X}$ chromosome from a non-heat-shocked larva. Throughout the length of the chromosome, the top half shows the RNA polymerase II and the bottom shows the B52. (B) A side-by-side comparison of several heat shock puffs stained with antibody to RNA polymerase II (left) and antibody to B52 (right). Each row shows the same puff, and the cytogenetic location is to the right of each pair of images. Maps are drawn to scale; black arrows indicate the heat shock genes responsible for the formation of each puff. (87A/C) IshHorowicz and Pinchin (1980); (67B) Costlow (1984), Ayme and Tissieres (1985); (95D) Holmgren et al. (1981); (transformant) YP1 transformant (which contains sequences from -89 to +62 of $h s p 70)$ from Kraus et al. (1988).

line 2 (SL2) cultured cells (Schneider 1972). Cells growing in culture media are irradiated with a UV light source resulting in the covalent cross-linking of protein to DNA. At the irradiation dose used, an average of one protein molecule is cross-linked per $60 \mathrm{~kb}$ of DNA /Gilmour and Lis 1985), such that the resulting proteinDNA adducts copurify with free DNA on a $\mathrm{CsCl}$ gradient. The detergent Sarkosyl and the high salt of the gradient disrupt noncovalent interactions and allow the separation of free protein from DNA. The DNA is then digested with restriction enzymes, and protein-DNA adducts are immunoprecipitated with antibody to B52. Samples are treated with a protease, and the DNA is analyzed by Southern blotting. The resolution of the in vivo distribution of B52 determined by this method is limited by the availability of restriction enzyme sites that can be cleaved under the conditions used to keep the protein-DNA adducts soluble.

Heat shock triggers the association of B52 with specific restriction fragments of the $87 \mathrm{~A}$ locus. In unstressed cells, no B52 is detected on these restriction fragments. In heat shock cells, however, B52 is crosslinked to the $10.5-\mathrm{kb}$ EcoRI fragment, which spans both of the heat shock genes found at the $87 \mathrm{~A}$ locus, but not to DNA fragments flanking the distal side of the $10.5-\mathrm{kb}$ fragment (Fig. 4). This locus was dissected further with other restriction enzymes, and the cross-linked B52 was localized more precisely to areas near the termination sites of both hsp70 genes (Fig. 5). The $3^{\prime}$ ends of these primary transcripts have been mapped to within 250 bases downstream of the SalI sites by determining the distribution of RNA polymerase II with UV cross-linking (Gilmour and Lis 1985; Gilmour et al. 1986). Therefore, B52 is present on the 3 ' ends of these genes and possibly on sequences immediately flanking them. No B52 is detected on the $5^{\prime}$ ends of the genes or in the intergenic region.

In Figure 5B, we suggest an alignment between the positions of B52 determined by UV cross-linking on nonpolytene chromosomes with those seen by immunofluorescence on the heat shock puff. Both assays show that B52 immediately flanks the RNA polymerases associated with the heat shock genes at the 87A locus (Figs. 3 and 5). Furthermore, the pair of B52 signals seen by UV cross-linking reside between the scs and scs' sites (Fig. 5) that have been proposed to be at the boundaries of the 87A puff (Udvardy et al. 1985; Kellum and Schedl 1991). The distance between the two B52 immunofluorescence signals on the $87 \mathrm{~A}$ puff is $\sim 1 \mu \mathrm{m}$, whereas the maximum and minimum distances between the two by UV crosslinking are 6.0 - and $3.6-\mathrm{kb}$ (equivalent to $2.0 \mu \mathrm{m}$ and 1.2 $\mu \mathrm{m}$ of B-form DNAl, respectively. Although the squashed chromosomes could be somewhat stretched relative to their native state, the DNA between the B52 brackets appears to be highly extended, approaching a packing ratio close to that of $\mathrm{B}$-form DNA.

The distribution of B52 at the 67B locus is similar to that at $87 \mathrm{~A}$ even though the organization of transcription units at each locus is quite different (Fig. 6). The 67B locus consists of seven developmentally regulated genes spread over $\sim 15 \mathrm{~kb}$. Four of the genes (hsp22, hsp23, hsp26, and hsp27) respond strongly to heat shock throughout development whereas the other three Igene 1 , gene 2, and gene 3) are strongly heat inducible only at certain times in development (Ayme and Tissieres 1985). In the SL2 cells used for UV cross-linking, transcription of genes 1,2, and 3 is not detected by nuclear run-on analysis in the presence or absence of heat shock (D.T. Champlin, T. O'Brien, and J.T. Lis, unpubl.). As with the 87A locus, no B52 was detected on the DNA near the heat shock genes at this locus in the absence of heat shock. During heat shock, B52 is recruited to regions flanking the outermost heat-induced genes. B52 is not found near every highly transcribed gene, for example, hsp23 and hsp26, even though hsp26 is similar in length 


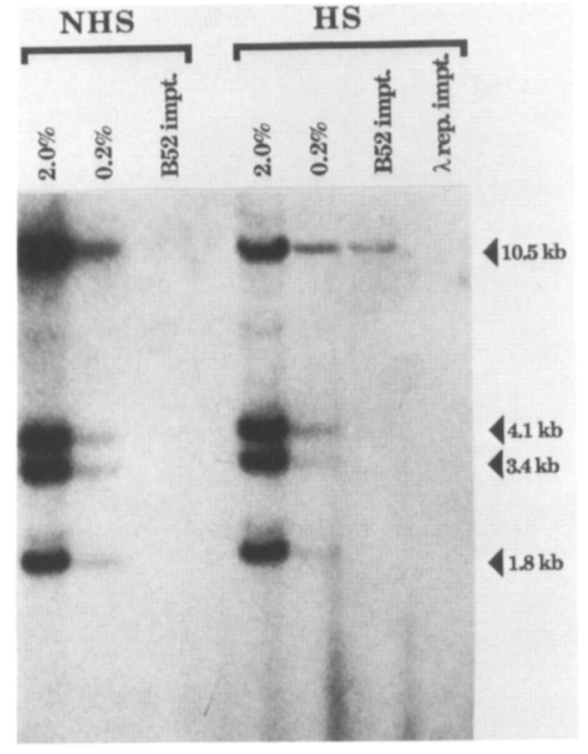

Figure 4. Identification of $\mathrm{B} 52$ at the $87 \mathrm{~A}$ heat shock locus in nonpolytene chromosomes by UV cross-linking. UV cross-linking of non-heat-shocked (NHS) and heat-shocked (HS) SL2 cells. DNA and protein-DNA complexes from UV-irradiated cells were restriction digested with EcoRI. DNA fragments were isolated following immunoprecipitation of protein-DNA adducts with monoclonal antibody to B52 (B52 impt.) or as a negative control with antibody to $\lambda$ repressor $(\lambda$ rep. impt.). Percentages of the total DNA sample used for immunoprecipitation are included to show the digestion pattern and to allow for quantitation $(2.0 \%$ and $0.2 \%)$. The Southern blot was probed with the 13.9-kb insert from $\lambda b D m 2$ (D. O'Connor and J.T. Lis, unpubl.), which spans the $87 \mathrm{~A}$ locus.

and heat-induced expression to hsp27 (Ayme and Tissieres 1985). Furthermore, B52 is found upstream of the hsp22 gene, suggesting that it is not simply involved in $3^{\prime}$-end formation. This is not surprising, as similar B52 signals are observed on both sides of puffs that contain single heat shock genes (Fig. 3B). It is interesting to note that gene 1 is located within the region bracketed by $\mathrm{B} 52$ during heat shock, but its level of transcription is not induced by heat shock in SL2 cells. Therefore, location of a gene within a region bracketed by B52 is not sufficient by itself to induce expression of the gene.

\section{Characterization of the B52 gene}

A $\lambda$ gt 11 expression library from Drosophila embryos (provided by U. Rosenberg and H. Jäckle) was screened with the B52 monoclonal antibody. Southern blot analysis revealed that all of the isolates obtained had portions of the same gene, and all were significantly shorter than the 1.6-kb mRNA detected by Northern blot analysis (Fig. 7). A second cDNA library (Salvaterra and Itakura 1985| was screened with the original clones by DNA hybridization at high stringency, and 24 positive samples were obtained. All had congruent restriction en- zyme maps, and 18 of the positive samples contained inserts of $\sim 1.6 \mathrm{~kb}$.

To confirm that we had cloned the gene corresponding to the puff border protein, we generated polyclonal rabbit antisera directed against a $\beta$-galactosidase-B52 fusion protein overexpressed in $E$. coli. The antisera obtained was purified over a trpE-B52 fusion protein affinity column, and the resulting antibody gave the same result as the original monoclonal antibody when used for indirect immunofluorescence of polytene chromosomes and UV cross-linking (data not shown). In addition, both antibodies identify a $52-\mathrm{kD}$ protein in SL2 cells and salivary glands by Western blot analysis (Fig. 7A).

B52 mRNA is present at all stages of Drosophila development that we examined (Fig. 7B). The B52 gene is located at the $87 \mathrm{~F}$ locus, and Southern blot analysis re-

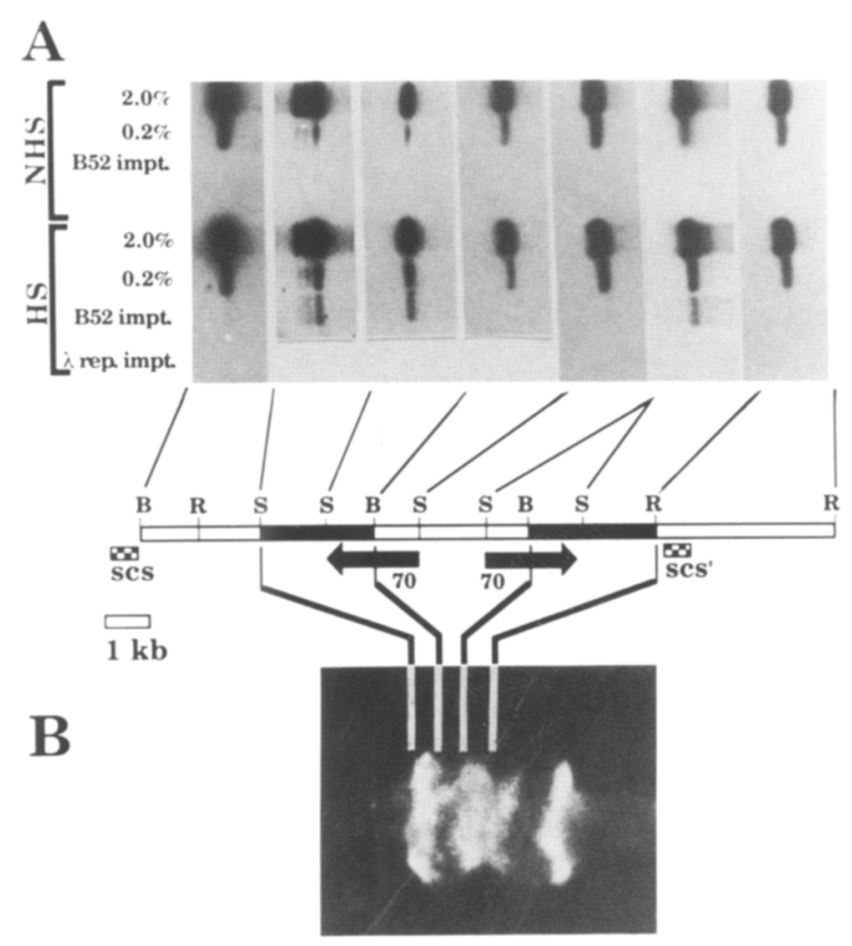

Figure 5. Distribution of $\mathrm{B} 52$ at the $87 \mathrm{~A}$ locus by UV crosslinking. $(A)$ A series of UV cross-linking experiments done as in Fig. 4 to further localize $\mathrm{B} 52$ on the $10.5-\mathrm{kb}$ EcoRI fragment identified in Fig. 4. The restriction map summarizes the observed distribution of B52 in heat-shocked cells; solid bars indicate DNA fragments that are cross-linked to B52 during heat shock; open bars indicate fragments that do not cross-link with B52 during heat shock. The restriction fragments examined are aligned in each case to the corresponding cross-linking result. Restriction sites used: (B) BamHI; (R) EcoRI; (S) Sall. The $5^{\prime}$ and $3^{\prime}$ ' $s p 70$ gene probes do not distinguish between the two copies of the gene whose positions are shown by black arrows. The scs and scs' regions (Udvardy et al. 1985) are indicated. Southern blot filters were probed with DNA fragments from $\lambda b D m 1$ and $\lambda \mathrm{bDm} 2$ (D. O'Connor and J.T. Lis, unpubl.). (B) Distribution of $\mathrm{B} 52$ at the $87 \mathrm{~A}$ locus on nonpolytene chromosomes aligned with the B52 distribution seen at this locus on polytene chromosomes. 


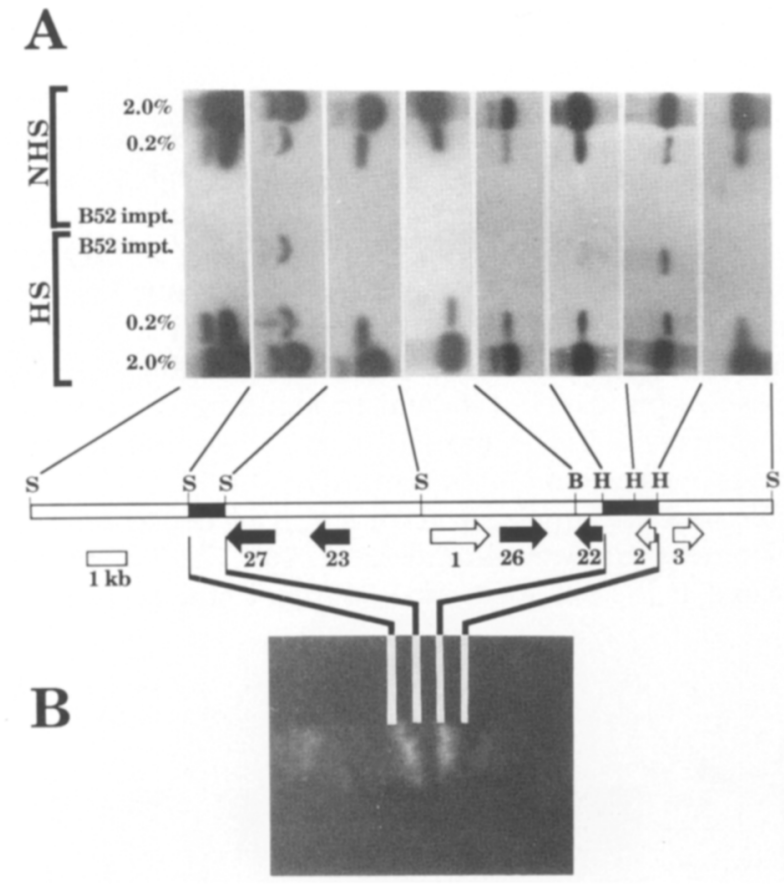

Figure 6. Distribution of B52 at the 67B locus by UV crosslinking. $(A)$ A series of UV cross-linking experiments done as in Fig. 4 to localize B52 at the 67B locus. The restriction map summarizes the observed distribution of B52 in heat-shocked cells. Solid bars indicate DNA fragments cross-linked to B52 during heat shock; open bars indicate fragments that do not cross-link with B52 during heat shock. The restriction fragments examined are aligned in each case to the corresponding cross-linking result. Restriction sites used: (B) BamHI; (H) HindIII; (S) SalI. Black arrows show positions of the four heat shock genes that are heat-inducible in SL2 cells; white arrows show positions of three genes that are not heat-inducible in these cells. Southern blot filters were probed with DNA fragments from $\lambda b D m 202$ (Costlow 1984) and cosmid c67B.RIC (D.T. Champlin and J.T. Lis). (B) Distribution of B52 at the 67B locus on nonpolytene chromosomes is aligned with the B52 distribution seen at this locus on polytene chromosomes.

vealed that a single copy of the gene is at this locus (Fig. 7C; data not shown).

Nucleotide sequencing of two of the cDNA clones identified an open reading frame of 346 codons (Fig. 8). An in-frame stop codon was found 15 nucleotides upstream of the first AUG, indicating that we have probably cloned the entire coding region of the $\mathrm{B} 52$ gene. The predicted protein has a molecular mass of only $43 \mathrm{kD}, 9$ $\mathrm{kD}$ less than measured on SDS-PAGE. However, a trpEB52 fusion protein also migrates more slowly on SDSPAGE than expected from its predicted molecular mass (data not shown). The B52 protein can be operationally divided into three regions separated by glycine-rich stretches. The first region contains strong similarity to an 80-amino-acid region found in a number of proteins that bind RNA and single-stranded DNA (Query et al. 1989). A 6/6 and a 7/8 match are found to the two most conserved sequences within this region, designated
RNP2 and RNP1 (Chan et al. 1989). The second region contains no identified sequence similarity to other known proteins. The third, carboxy-terminal, region of the protein is dominated by a regular repeat of serine and arginine or lysine. Consequently, the protein is predicted to be very basic; this has been confirmed by nonequilibrated $\mathrm{pH}$-gradient electrophoresis (O'Farrell et al. 1977; data not shown). The abundance of serine and arginine is reminiscent of protamines, which displace histones during the condensation of sperm chromatin (Warrant and Kim 1978). Similarly organized repeats of serine and arginine are found in transformer (Boggs et al. 1987) and suppressor-of-white-apricot (Chou et al. 1987), which were identified genetically to affect RNA splicing of the doublesex gene and the white-apricot allele, respec-

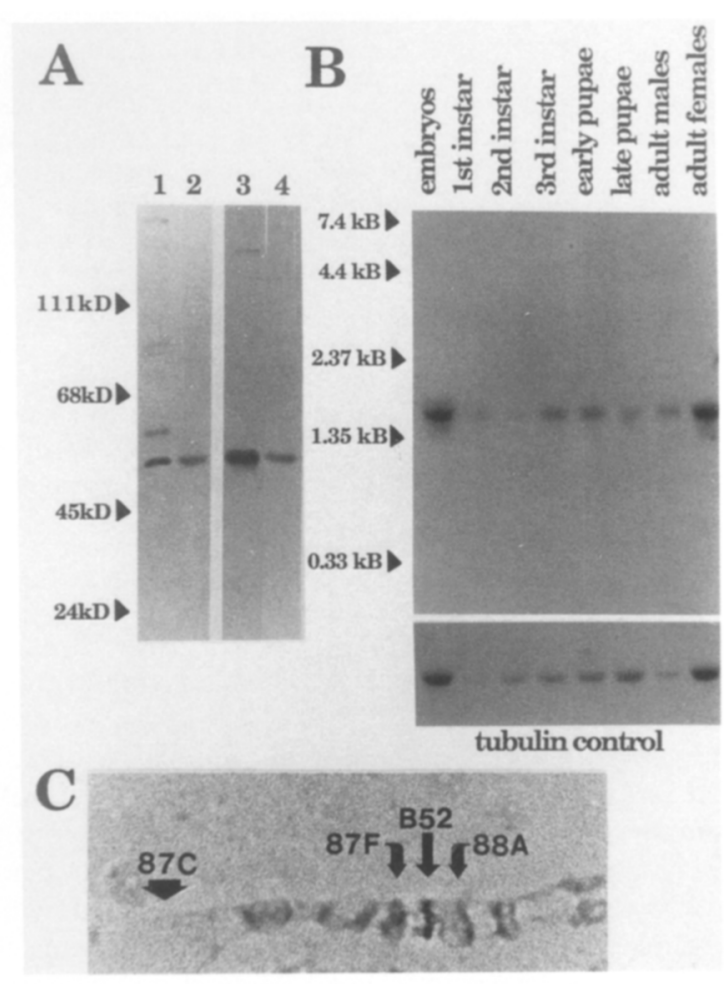

Figure 7. Characterization of the expression and genomic location of the B52 gene. (A) Western blot analysis of salivary glands from 10 third-instar larvae (lanes 1,3 ) or total cell lysates from $2 \times 10^{6} \mathrm{SL} 2$ cells (lanes 2,4). B52 protein was detected using the monoclonal antibody (lanes 1,2 ) or affinity-purified polyclonal antibody (lanes 3,4$)$. (B) Northern blot analysis of the B52 mRNA isolated from larval, pupal, and adult stages. The poly $(\mathrm{A})^{+}$RNA from $D$. melanogaster was isolated as described previously, and $\sim 5 \mu \mathrm{g}$ was loaded per lane (DiBenedetto et al. 1987). The filter-bound RNAs were hybridized with radiolabeled B52 cDNA. The B52 RNA signals should be compared relative to the tubulin standard (DiBenedetto et al. 1987). (C) In situ hybridization of the B52 gene to Drosophila salivary gland chromosomes. The hybridization and detection with biotin-labeled B52 cDNA was done as described previously (Simon et al. 1985b). The portion of chromosome 3 shown is from a heatshocked larva, and the diffuse $87 \mathrm{C}$ heat shock puff is labeled. 


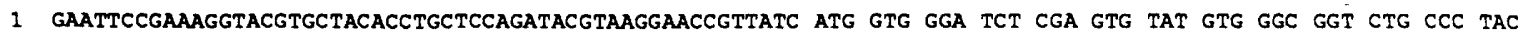
ECORI END Met Val Gly Ser Arg Val Tyr Val Gly Gly Leu Pro Tyr $\bullet \bullet \bullet \bullet$

94 GGA GTG CGC GAG CGC GAT TTG GAG CGC TTT TTC AAA GGC TAC GGC CGC ACA CGC GAC ATC CTC ATC AAA AAT GGC TAC GGC

14 Gly Val Arg Glu Arg Asp Leu Glu Arg Phe Phe Lys Gly Tyr Gly Arg Thr Arg Asp Ile Leu Ile Lys Asn Gly Tyr Gly

175 TTT GTG GAA ITC GAA GAC TAT CGT GAT GCC GAC GAT GCC GTC TAT GAA CTG AAT GGC AAA GAG CTG CTT GGC GAA CGT GTG

41 Phe Val Glu phe Glu Asp Tyr Arg Asp Ala Asp Asp Ala Val Tyr Glu Leu Asn Gly Lys Glu Leu Leu Gly Glu Arg Val - $\bullet$

256 GTT GTT GAA CCC GCC AGG GGT ACC GCT CGT GGC AGC AAC CGC GAC CGC TAC GAC GAT CGA TAT GGT GGT CGG CGG GGG GGC

68 Val Val Glu pro Ala Arg Gly Thr Ala Arg Gly Ser Asn Arg Asp Arg Tyr Asp Asp Arg Tyr Gly Gly Arg Arg Gly Gly

337 GGG GGC GGT CGT TAC AAC GAA AAA AAC AAA AAT TCC AGA TCA TCC TCT CGT TAT GGC CCA CCG TTG CGC ACT GAG TAC CGA

95 Gly Gly Gly Arg Tyr Asn Glu Lys Asn Lys Asn Ser arg Ser Ser Ser Arg Tyr Gly Pro Pro Leu Arg Thr Glu Tyr Arg

418 CTG ATT GTG GAG AAT TTG TCT AGC CGC GTT AGC TGG CAG GAT CTC AAG GAT TAC ATG CGC CAG GCT GGC GAG GTC ACC TAT

122 Leu Ile Val Glu Asn Leu Ser Ser Arg Val Ser Trp Gln Asp Leu Lys Asp Tyr Met Arg Gin Ala Gly Giu Val Thr Tyr

499 GCC GAT GCC CAC AAG CAG CGT CGC AAT GAG GGC GTG GTT GAG TTC GCC TCG TTG TCG GAC ATG AAG ACG GCC ATT GAG AAG

149 Ala Asp Ala His Lys Gln Arg Arg Asn Glu Gly Val Val Glu phe Ala Ser Leu Ser Asp Met Lys Thr Ala Ile Giu Lys

580 TTG GAT GAC ACC GAG CTA AAC GGC AGG CGG ATC CAC TTG GTC GAG GAT CGT CGC GGA GGA GCC AGC GGT GGT GGC GGC GGC

176 Leu Asp Asp Thr Glu Leu Asn Gly Arg Arg Ile His Leu Val Glu Asp Arg Arg Giy Gly Ala Ser Gly Gly Gly Giv Giy

661 AGC GGA CGT GGA CGC TCC CGT TCG TCC AGC TCC CGA TCG CGC TCC CGA TCC CGC AGG CGT TCT CGC TCT CGC CGC TCG ACG

203 Ser gly Arg Gly Arg Ser Arg Ser Ser Ser Ser Arg Ser Arg Ser Arg Ser Arg Arg Arg Ser Arg Ser Arg Arg Ser Thr

742 CAC TCG CGC TCC AAG TCT CGC AGC CGC TCC AAA TCT CGT GGC GGA CGC TCC AAG TCC AAG TCG CCA GTC AAG TCT CGT TCT

230 H1s Ser Arg Ser Lys Ser Arg Ser Arg Ser Lys Ser Arg Gly Gly Arg Ser Lys Ser Lys Ser Pro Val Lys Ser Arg Ser

823 CGC TCC CGC TCG GCA TCC AAC AAA TCG CGT GAC GTG TCC AAG TCA AAG TCC CAC TCC CGC ACC GCT CCC GTT TCG CCC AAA

257 Arg Ser Arg Ser Ala Ser Asn lys Ser Arg Asp Val Ser Lys Ser Lys Ser his Ser Arg Thr Ala pro Val Ser pro Lys

904 CGT GAG CGT GAC TCC CGT TCC CGA TCG CGG TCC GTC TCG AAG CGC GAG TCC CGT TCC CGC TCA CGT TCC AAG TCC ATC CAC

284 Arg Glu Arg Asp Ser Arg Ser Arg Ser Arg Ser Val Ser Lys Arg Glu Ser Arg Ser Arg Ser Arg Ser Lys Ser Ile His

985 CGC GAC TCC CGT TCA CGC CCG CCA ACG GTG TCG TTC AAA AGC TCG TTC TAT AAG TTC ACT ACA ATG CCT TTC TTT TGC AGC

311 Arg Asp Ser Arg Ser Arg Pro Pro Thr Val Ser phe lys Ser Ser phe Tyr Lys Phe Thr Thr Met pro Phe Phe Cys Ser

1066 GAT CGA TCC GCA TCG GCT GAA AAC AAG TCG CGT TCG CGC TCA CGC TCC CGT TCG GCC TCG CCC AAA AAT GGA AAC GCC TCA

338 Asp Arg Ser Ala Ser Ala Glu Asn Lys Ser Arg Ser Arg Ser Arg Ser Arg Ser Ala Ser Pro Lys Asn Gly Asn Ala Ser

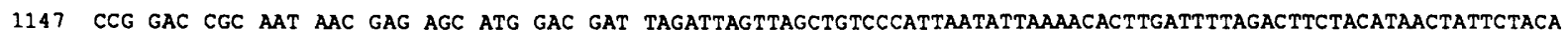

365 Pro Asp Arg Asn Asn Glu Ser Met Asp Asp END

1244 AACATCAACACTCAGACACCACCCCGACTACAACCAAAACTTAATGAATATGAAATCATACCAATCCACCACTGTCGGGGCCTCGTCATTTTCTTTTAAATATATAA 1351 CGACAATTTGTCCTAAGTCTTGTTGTGGGACAAGGGACATGGGCTGGAAGATGACAGGGATTGGCTGAATGTGCCGATTTGGATTCAAGGAGTACATATAGGCCACG

1458 AAATTACTGCGACTTGTACACCGGAACACTTAAAATTGTCTATACAATGAGTAATTTCAATAAATACGGATAAATTTAAACTAT POIYA

Figure 8. Nucleotide sequence and predicted primary amino acid sequence from the large open reading frame of the B52 cDNA. Nucleic acid and amino acid sequences are numbered at left. The in-frame termination codon upstream of the predicted initiation site is designated END. Glycine-rich regions are underscored. The matches to the RNA-binding consensus (Chan et al. 1989) are underscored with solid circles. The EcoRI site used to create fusion proteins is underscored.

tively. The alternative splice event regulated by transformer and suppressor-of-white-apricot may be at the level of splice site selection or poly(A) site selection (McKeown 1990), or transcription termination.

The presence of the RNA-binding domain and the highly basic repeat in B52 provides two potential sites for contacting nucleic acid. B52 has sequence similarity with some RNA-binding proteins such as those found in small nuclear ribonucleoproteins (snRNPs) and heterogenous RNPs (hnRNPs), but the distributions of snRNPs (Martin et al. 1987; S. Amero, pers. comm.) and hnRNPs (Christensen et al. 1981; Risau et al. 1983) are like that of RNA polymerase II on activated heat shock loci. Although B52 may bind RNA, we find that the protein is not extracted from nuclei by RNase treatment (see Materials and methods/. Likewise, RNase treatment of cross-linked protein-DNA complexes prior to immunoprecipitation does not affect the anti-B52 precipitation of specific restriction fragments seen in Figure 5 (see Materials and methods|. Therefore, the association of B52 with the specific regions of chromatin detected here does not appear to be through interactions with the RNA transcripts of these regions.

\section{Discussion}

The B52 protein is associated with transcriptionally active chromatin, but it has a distribution that is different from any protein described previously. On polytene chromosomes, its distribution is similar to that of RNA polymerase II, and similar changes in the distributions of both proteins occur upon induction of the heat shock genes. At most loci, the two proteins appear to coincide; but on the largest heat shock-induced puffs, B52 occupies positions that are clearly distinct from that of RNA polymerase II. At these loci, B52 brackets the RNA poly- 
merase II signal symmetrically. The recruitment of B52 to heat shock loci upon activation of the heat shock genes is also observed in nonpolytene chromosomes by UV cross-linking, and the high resolution afforded by this technique confirms that B52 is not evenly distributed on transcription units but, instead, parenthetically defines transcriptionally active regions. When several highly transcribed genes are clustered, B52 does not flank each transcription unit but, instead, borders a domain that encompasses the entire transcriptionally active region.

Whereas high levels of transcription can perturb chromatin structure beyond the limits of the gene (van Holde 1988 |, the presence of more than one highly transcribed gene at a particular chomosomal location may lead to the merging of these regions of altered chromatin structure. This would create a larger domain encompassing a set of discrete transcription units. Consistent with this hypothesis is the observation that a single chromosomal puff often contains multiple active genes. B52 brackets these genes, presumably recognizing the boundaries of a higher order structure encompassing all of the active transcription units. B52 also brackets clusters of transcriptionally active genes in a similar manner in nonpolytene cells, indicating that such higher order domains are not restricted to polytene chromosomes. In addition to the endogenous heat shock loci, B52 forms brackets at new heat shock puffs produced in transgenic lines, suggesting that the distribution may not be due to binding a specific DNA sequence. The data presented here, however, do not discriminate between an active or passive role for the B52 protein in establishing these domains.

B52 is found near the boundaries of the general nuclease-sensitive regions of chromatin at the heat shockactivated loci $67 \mathrm{~B}$ and $87 \mathrm{~A}$. Activation of transcription results in a general increase in sensitivity to a number of nucleases spanning the region bracketed by $\mathrm{B} 52$ at the 87A locus (Han et al. 1984; Udvardy and Schedl 1984). Heat shock causes little change in nuclease sensitivity outside of the bracketed region except for changes within two nuclease hypersensitive regions (scs and scs') that flank the locus (Udvardy et al. 1985). The boundaries of general nuclease sensitivity and B52 localization do not correspond to these scs regions or to the single scaffoldattached region (SAR) found at the 87A locus in LISextracted nuclei (Mirkovitch et al. 1984).

At the 67B locus, B52 is also found near the borders of the region that exhibit a general increase in DNase I sensitivity resulting from activation of the four small heat shock genes (Costlow 1984). Although differences between the transcribed and nontranscribed DNA at the 67B locus have been detected with MPE-Fe(II) (Cartwright and Elgin 1986), no significant difference in general DNase I sensitivity was seen between the transcribed and nontranscribed DNA spanning the region bracketed by B52 (Costlow 1984). Thus, B52 does not bracket the individual transcription units but, rather, the entire nuclease-sensitive domain.

The bulk of topoisomerase I and II cleavages created during heat shock-induced transcription are within the region bounded by B52. Topoisomerase I is recruited to heat shock loci during heat shock (Fleischmann et al. 1984; Gilmour et al. 1986; Gilmour and Elgin 1987), but it is not uniformly distributed within these active loci. Instead, topoisomerase I is found to associate predominantly with the transcribed regions, and little or no topoisomerase I is found on the nontranscribed intergenic regions. Most heat shock-induced topoisomerase II cleavage sites at the $87 \mathrm{~A}$ locus are also within the region bracketed by B52 (Rowe et al. 1986; Udvardy and Schedl 1986), although Rowe et al. (1986) reported some cleavage at sites outside of this region, in contrast with Udvardy and Schedl (1986).

It is interesting to note that gene 1 is located within the region bracketed by B52 during heat shock (Fig. 6), but its transcription is not detected in heat-shocked SL2 cells. Therefore, the presence of a gene within a region bracketed by $\mathrm{B} 52$ is not sufficient by itself to induce its expression. However, when gene 1 expression is induced by ecdysone during Drosophila development, heat shock greatly increases the expression of the gene to a level comparable to that of other major heat shock genes (Ayme and Tissieres 1985). This high level of expression of gene 1 may be due to heat shock factor binding to the locus and further stimulating the ecdysone-activated gene 1 promoter. Alternatively, transcription of the ecdysone-stimulated gene 1 may be facilitated by its inclusion in the heat shock-induced domain bracketed by B52.

We have found B52 associated with numerous interbands and puffs during normal development but have observed distinct bracketing only on heat shock puffs. The increased resolution afforded by the highly decondensed state of heat shock puffs provides a possible explanation for why bracketing is not observed at most of these developmental loci. However, B52 does not clearly bracket even those developmental puffs that are as large as the heat shock puffs. Puff size is determined by a combination of transcription frequency and transcript length (Simon et al. 1985a). Some developmental puffs contain long transcription units (Thummel et al. 1990), but many of these appear to be transcribed at a frequency lower than that of fully induced, major heat shock genes (Jamrich et al. 1977). The very high transcription frequency within the heat shock puffs may be responsible for the ease of detecting bracketing. On moderately expressed genes, nucleosomes (McKnight et al. 1978) and even 30-nm beads (Bjorkroth et al. 1988) reappear between succesive RNA polymerases. On very highly expressed genes, nucleosomes remain in an altered configuration, and the 30-nm fiber does not form (see introductory section). We suggest that B52 participates in the condensation or decondensation of chromatin, perhaps at the level of the $30-\mathrm{nm}$ fiber. In the case of the highly expressed heat shock genes, such changes in chromatin are restricted to the boundaries of the highly active domain; whereas at other moderately active loci, these changes occur throughout the transcribed region. Indeed, preliminary experiments indicate that when the heat shock genes are induced to only moderate levels, B52 is 
found by UV cross-linking to be associated within the region that it normally brackets when the genes are fully induced (D.T. Champlin and J.T. Lis, unpubl.).

\section{Materials and methods}

Antibodies

The generation of a large set of monoclonal antibodies, including an antibody to B52 (antibody designated Bv32 in this series), has been described (Frasch 1985; Frasch and Saumweber 1989). The B52 antibody was obtained (from nuclei treated with RNase) by using a fraction of the soluble chromatin proteins stripped with $450 \mathrm{~mm} \mathrm{NaCl}$ for immunization. Polyclonal antiserum directed against B52 was generated in rabbits against a LacZ-B52 fusion protein overexpressed in $E$. coli containing the pUR32.18 plasmid. Purification of the resulting antisera over an affinity column containing the TrpE-B52 fusion protein was done as described (Rio et al. 1986). Affinity-purified polyclonal antiserum directed against RNA polymerase II was generated in rabbits with a LacZ-RNA polymerase II fusion protein overexpressed in $E$. coli that contained a portion of the large subunit of RNA polymerase II encoded by exon 2 and purified over a column containing the fusion protein (E. Wong and J. Lis, unpubl.).

\section{Indirect immunofluorescence}

The $A d h^{f n 6} c n$;ry502 strain (Simon et al. 1985a) and transformant lines derived from it were used for indirect immunofluorescence of salivary gland polytene chromosomes, as described (Risau et al. 1983), with the following modifications. Heat shock was done for $30 \mathrm{~min}$ at $36.5^{\circ} \mathrm{C}$. The dissection and fixation buffers all contained $1 \%$ Triton. Following squashing, the slides were frozen in liquid $\mathrm{N}_{2}$ and stored in ethanol at $4^{\circ} \mathrm{C}$ for up to 2 days. Slides were rehydrated with TBS $[10 \mathrm{mM}$ Tris- $\mathrm{HCl}$ (pH 7.4), $150 \mathrm{~mm} \mathrm{NaCl}$ ] and incubated under coverslips in a humid chamber for $1 \mathrm{hr}$ each with the following: TBS $/ 10 \%$ goat serum, TBS $/ 10 \%$ goat serum with $1: 200$ B52 monoclonal and $1: 50$ affinity-purified RNA polymerase II polyclonal antibodies, and TBS $/ 10 \%$ goat serum with $1: 200$ fluorochrome-conjugated goat secondary antibodies (Cappel). After photography, slides were stained for $5 \mathrm{~min}$ in TBS with a $1: 10^{5}$ dilution of 1 $\mathrm{mg} / \mathrm{ml}$ of Hoechst 33258 (Sigma) and re-examined. Photography was done using a Zeiss Universal fluorescence microscope and M63 camera with Kodak TMZ-P3200 film.

\section{UV cross-linking}

UV cross-linking was done as described (Gilmour and Lis 1985), with the following modifications: Each sample had $10^{8}$ SL2 cells. In the restriction digests $1 \%$ Triton replaced NP-40. Proteinase $\mathrm{K}$ digestion was done at $65^{\circ} \mathrm{C}$ for $5 \mathrm{hr}$. Gels were blotted to GeneScreen Plus (NEN) and probed according to the manufacturer. As determined by titration with ${ }^{35} \mathrm{~S}$-labeled LacZ-B52 fusion protein, $10 \mu \mathrm{l}$ of mouse ascites fluid or $75 \mu \mathrm{l}$ of affinitypurified rabbit polyclonal antibody was used for each sample. Immunoprecipitations with monoclonal antibody were followed by $35 \mu$ l of rabbit anti-mouse IgG. In either case, $100 \mu \mathrm{l}$ of Pansorbin (CalBiochem) was then used to immunoprecipitate the antibodies. All antibody incubations took place for $1 \mathrm{hr}$. With the monoclonal antibody, complexes were washed at $\mathrm{pH}$ 8.0.

For RNase treatment prior to immunoprecipitation, dialyzed samples were treated with $0.5 \mu \mathrm{g} / \mathrm{ml}$ of protease-free RNase (CalBiochem) for $2 \mathrm{hr}$ at $37^{\circ} \mathrm{C}$. This incubation was sufficient to completely degrade a radiolabeled SP6 transcript added to con- trol samples. The pattern of hybridization was indistinguishable from that seen in Figure 5 in the absence of RNase treatment.

\section{Cloning and sequencing}

Screening of the $\lambda g t 11$ library was done as described (Young and Davis 1983). Positive clones were subcloned as KpnI-SalI fragments or EcoRI fragments, sequenced, and then subcloned into pUC19 to give p15.3, which contains the entire sequence as reported here. Sequencing was done by the dideoxy chain-termination method using Sequenase (U.S. Biochemical) according to the manufacturer's instructions. Plasmids for fusion protein synthesis were made by subcloning the EcoRI fragment of the B52 cDNA starting at the endogenous EcoRI site into pUR289 (Ruther and Muller-Hill 1983) resulting in plasmids pUR32.18 and pATH3.32 (Dieckmann and Tzagoloff 1985). Induction and partial purification of such fusion proteins have been described (Spindler et al. 1984; Rio et al. 1986). Additional DNA used to probe the B52 distribution at the 67B locus was subcloned from the c67B.RlC cosmid clone isolated from the iso-1 genomic cosmid library provided by John Tamkun.

\section{Western blots}

Proteins were electrophoresed on $8 \%$ SDS-polyacrylamide gels and electrophoretically transferred to nitrocellulose (Schleicher \& Schuell; Towbin et al. 1979|. B52 was detected by sequential incubations of the blots with a $1: 1000$ dilution of monoclonal or affinity-purified polyclonal antibody to B52, biotinylated goat anti-mouse or anti-rabbit IgG, streptavidin-horseradish peroxidase conjugate, and 4-chloro-1-napthol. When the monoclonal antibody was used, $0.05 \%$ Tween 20 (Sigma) was added to incubations and washes.

\section{Acknowledgments}

We thank Dr. F. Bonhoeffer for his encouragement and support and Monika Wild for technical assistance. We thank Mike Goldberg for the developmental Northern blot. This work was supported by National Institutes of Health grant GM40918 to J.L., and National Research Service Award GM 07273 and a ARO Biotechnology Predoctoral Fellowship to D.C.

The publication costs of this article were defrayed in part by payment of page charges. This article must therefore be hereby marked "advertisement" in accordance with 18 USC section 1734 solely to indicate this fact.

\section{Note added in proof}

Sequence data described in this paper have been submitted to the EMBL/GenBank Data Libraries.

Two reports of the primary structure of an alternative splicing factor from humans appeared when our article was in press [Ge, H., P. Zuo, and J.L. Manley. (1991). Cell 66: 373-382; Krainer, A.R., A. Mayeda, D. Kozak, and G. Binns. (1991). Cell 66: 383394]. This factor, called ASF or SF2, affects the utilization of alternative $5^{\prime}$-splice sites in vitro. The homology between ASF/ SF2 and B52 is striking. A sequence comparison of B52 with ASF/SF2 using the GCG package (University of Wisconsin) shows that these proteins have $67 \%$ similarity and $52 \%$ identity over 233 amino acids of the 248-amino-acid ASF/SF2 protein. $\mathrm{B} 52$ has a predicted molecular mass that is $15.3 \mathrm{kD}$ larger than the 27.7-kD ASF/SF2, resulting mainly from an extended SerArg-repeat domain. If the sole in vivo function of ASF/SF2 is to 
modulate $5^{\prime}$-splice site utilization, and if B52 is indeed a functional homolog, then the observed associations of B52 with transcriptionally active chromosomal DNA are intriguing. Perhaps the accumulation of B52 at active loci via its interaction with chromatin positions it to alter splicing if the appropriate target pre-mRNAs are present. This presents the possibility for interplay between mechanisms of transcriptional regulation and splicing control.

\section{References}

Ayme, A. and A. Tissieres. 1985. Locus 67B of Drosophila melanogaster contains seven, not four, closely related heat shock genes. EMBO I. 4: 2949-2954.

Bjorkroth, B., C. Ericsson, M.M. Lamb, and B. Daneholt. 1988. Structure of the chromatin axis during transcription. Chromosoma 96: 333-340.

Boggs, R. T. and McKeown, M. 1987. Regulation of sexual differentiation in Drosophila melanogaster via alternative splicing of RNA from the transformer gene. Cell 50: 739747.

Brill, S.J., S. DiNardo, K. Voelkel-Meiman, and R. Sternglanz. 1987. Need for DNA topoisomerase activity as a swivel for DNA replication for transcription of ribosomal RNA. $\mathrm{Na}$ ture 326: 414-416.

Burtis, K.C., C.S. Thummel, C.W. Jones, F.D. Karim, and D.S. Hogness. 1990. The Drosophila 74EF early puff contains E74, a complex ecdysone-inducible gene that encodes two etsrelated proteins. 61: 85-99.

Cartwright, I.L. and S.C.R. Elgin. 1986. Nucleosomal instability and induction of new upstream protein-DNA associations accompany activation of four small heat shock protein genes in Drosophila melanogaster. Mol. Cell. Biol. 6: 779-791.

Chan, E.K.L., K.F. Sullivan, and E.M. Tan. 1989. Ribonucleoprotein SS-B/La belongs to a protein family with consensus sequences for RNA-binding. Nucleic Acids Res. 17: 2233-.

Chou, T.-B., Z. Zachar, and P.M. Bingham. 1987. Developmental expression of a regulatory gene is programmed at the level of splicing. EMBO I. 6: 4095-4104

Christensen, M.E., and S.C.R. Elgin. 1981. Distribution studies on polytene chromosomes using antibodies directed against hnRNP. J. Cell Biol. 90: 18-24.

Costlow, N. 1984. "Chromatin structure of Drosophila heat shock genes in Drosophila and in yeast as probed with DNase I." Ph.D. thesis, Comell University, Ithaca.

Csordas, A. 1990. On the biological role of histone acetylation. Biochem. I. 265: 23-38.

DiBenedetto, A.J., D.M. Lakich, W.D. Kruger, J.M. Belote, B.S. Baker, and M.F. Wolfner. 1987. Sequences expressed sex-specifically in Drosophila melanogaster adults. Dev. Biol. 119: 242-251.

Dieckmann, C.L. and A. Tzagoloff. 1985. Assembly of the mitochondrial membrane system. J. Biol. Chem. 260: 15131520.

Eisenberg, J.C., I.L. Cartwright, G.H. Thomas, and S.C.R. Elgin. 1985. Selected topics in chromatin structure. Annu. Rev. Genet. 19: 485-536.

Fleischmann, G., G. Pflugfelder, E.K. Steiner, K. Javaherian, G.C. Howard, J.C. Wang, and S.C. Elgin. 1984. Drosophila DNA topoisomerase $I$ is associated with transcriptionally active regions of the genome. Proc. Natl. Acad. Sci. 81: 6958-6962.

Frasch, M. 1985. "Charakterisierung chromatinassoziierter kernproteine von Drosophila melanogaster mit hilfe monoklonaler antikorper." Ph.D. thesis, Eberhard-Karls-Universi- tat, Tübingen, Germany.

Frasch, M. and H. Saumweber. 1989. Two proteins from Drosophila nuclei are bound to chromatin and are detected in a series of puffs on poytene chromosomes. Chromosoma 97: 272-281.

Gilmour, D.S. and S.C.R. Elgin. 1987. Localization of specific topoisomerase I interactions within the transcribed region of active heat shock genes by using the inhibitor camptothecin. Mol. Cell. Biol. 7: 141-148.

Gilmour, D.S. and J.T. Lis. 1985. In vivo interactions of RNA polymerase II with genes of Drosophila melanogaster. Mol. Cell. Biol. 5: 2009-2018.

- 1986. RNA polymerase II interacts with the promoter region of the noninduced hsp70 gene in Drosophila melanogaster cells. Mol. Cell. Biol. 6: 3984-3989.

Gilmour, D.S., G. Pflugfelder, J.C. Wang, and J.T. Lis. 1986. Topoisomerase I interacts with transcribed regions in Drosophila cells. Cell 44: 401-407.

Han, S., A. Udvardy, and P. Schedl. 1984. Transcriptionally active chromatin is sensitive to Neurospora crassa and SI nucleases. I. Mol. Biol. 179: 469-496.

Heller, R.A., E.R. Shelton, V. Dietrich, S.C.R. Elgin, and D. Brutlag. 1986. Multiple forms and cellular localization of Drosophila DNA topoisomerase II. J. Biol. Chem. 261: 8063-8069.

Holmgren, R., V. Corces, R. Morimoto, R. Blackman, and M. Meselson. 1981. Sequence homologies in the $5^{\prime}$ regions of four Drosophila heat-shock genes. Proc. Natl. Acad. Sci. 78: 3775-3778.

Ish-Horowicz, D. and S.M. Pinchin. 1980. Genomic organization of the $87 \mathrm{~A} 7$ and $87 \mathrm{C} 1$ heat-induced loci of Drosophila melanogaster. J. Mol. Biol. 142: 231-245.

Jamrich, M., A.L. Greenleaf, and E.K.F. Bautz. 1977. Localization of RNA polymerase in polytene chromosomes of Drosophila melanogaster. Proc. Nat1. Acad. Sci. 74: 2079-2083.

Kellum, R. and P. Schedl. 1991. A position-effect assay for boundaries of higher order chromosomal domains. Cell 64: 941-950.

Kraus, K.W., Y. Lee, J.T. Lis, and M.F. Wolfner. 1988. Sex-specific control of Drosophila melanogaster yolk protein 1 gene expression is limited to transcription. Mol. Cell. Biol. 8: 4756-4764.

Martin, T.E., S.A. Monsma, J.M.-J. Romac, and G.P. Leser. 1987. The intranuclear states of snRNP complexes. Mol. Biol. Rep. 12: $180-181$

McKeown, M. 1990. Regulation of alternative splicing. In $\mathrm{Ge}$ netic engineering, vol. 12 (ed. J.K. Setlow), pp. 139-181. Plenum Press, New York.

McKnight, S.L., M. Bustin, and O.L. Miller, Jr. 1978. Electron microscopic analysis of chromosome metabolism in the Drosophila melanogaster embryo. Cold Spring Harbor Symp. Quant. Biol. 42: 741-754.

Mirkovitch, J., M.-E. Mirault, and U.K. Laemmli. 1984. Organization of the higher-order chromatin loop: Specific DNA attachment sites on nuclear scaffold. Cell 32: 223-232.

Nacheva, G.A., D.Y. Guschin, O.V. Preobrazhenskaya, V.L. Karpov, K.K. Ebralidse, and A.D. Mirzabekov. 1989. Change in the pattern of histone binding to DNA upon transcriptional activation. Cell 58: 27-36.

O'Farrell, P.A., H.M. Goodman, and P.H. O'Farrell. 1977. High resolution two-dimensional electrophoresis of basic as well as acidic proteins. Cell 12: 1133-1142.

Query, C.C., R.C. Bentley, and J.D. Keene. 1989. A common RNA recognition motif identified within a defined U1 RNA binding domain of the 70K U1 snRNP protein. Cell 57: 89101.

Rio, D.C., F.A. Laski, and G.M. Rubin. 1986. Identification and 
immunochemical analysis of biologically active Drosophila P element transposase. Cell 44: 21-32.

Risau, W., P. Symmons, H. Saumweber, and M. Frasch. 1983. Nonpackaging and packaging proteins of hnRNA in Drosophila melanogaster. Cell 33: 529-541.

Rowe, T.C., J.C. Wang, and L.F. Liu. 1986. In vivo localization of DNA topoisomerase II cleavage sites on Drosophila heat shock chromatin. Mol. Cell. Bio. 6: 985-992.

Ruther, U. and B. Muller-Hill. 1983. Easy identification of cDNA clones. EMBO I. 2: 1791-1794.

Salvaterra, I.N. and K. Itakura. 1985. Drosophila Inf. Serv. 61: 89.

Schneider, I. 1972. Cell lines derived from late embryonic stages of Drosophila melanogaster. I. Embryol. Exp. Morphol. 27: 353-365.

Silver, L.M. and S.C.R. Elgin. 1976. A method for determination of the in situ distribution of chromosomal proteins. Proc. Natl. Acad. Sci. 73: 423-427.

1978. Immunological analysis of protein distributions in Drosophila polytene chromosomes. In The cell nucleus, chromatin part $B$ (ed. H. Busch), vol. 5, pp. 215-262. Academic Press, New York.

Simon, J.A., C.A. Sutton, R.B. Lobell, R.B. Glaser, and J.T. Lis 1985a. Determinants of heat shock-induced chromosome puffing. Cell 40: 805-817.

Simon, J.A., C.A. Sutton, and J.T. Lis. 1985b. Localization and expression of transformed DNA sequences within heat shock puffs of Drosophila melanogaster. Chromosoma 93: $26-30$.

Solomon, M.J., P.L. Larsen, and A. Varshavsky. 1988. Mapping protein-DNA interactions in vivo with formaldehyde: Evidence that histone $\mathrm{H} 4$ is retained on a highly transcribed gene. Cell 53: 937-947.

Spindler, K.R., D.S.E. Rosser, and A.J. Berk. 1984. Analysis of adenovirus transforming proteins from early region $\mathrm{IA}$ and $1 \mathrm{~B}$ with antisera to inducible fusion antigens produced in Escherichia coli. I. Virol. 49: 132-141.

Thomas, G.H. and S.C.R. Elgin. 1988. Protein/DNA architecture of the DNase I hypersensitive region of the Drosophila hsp26 promoter. EMBO /. 7: 2191-2201.

Towbin, H., T. Staehlin, and J. Gordon. 1979. Electrophoretic transfer of proteins from polyacrylamide gels to nitrocellulose sheets; procedure and some application. Proc. Natl. Acad. Sci. 76: 4350-4354.

Udvardy, A. and P. Schedl. 1984. Chromatin organization of the 87A7 Heat shock locus of Drosophila melanogaster. I. Mol. Biol. 172: 385-403.

. 1986. Topoisomerase II cleavage in chromatin. J. Mol. Biol. 191: 231-246.

Udvardy, A., E. Maine, and P. Schedl. 1985. The 87A7 chromomere: Identification of novel chromatin structures flanking the heat shock locus that may define the boundaries of higher order domains. J. Mol. Biol. 185: 34l-358.

van Holde, K. E. 1988. Chromatin. Springer-Verlag, Berlin.

Warrant, R.D. and S.-H. Kim. 1978. $\alpha$-Helix-double helix interaction shown in the structure of a protamine-transfer RNA complex and a nucleoprotamine model. Nature 271: 130135.

Wu, C. 1984. Two protein-binding sites in chromatin implicated in the activation of heat-shock genes. Nature 309: 229-234.

Young, R.A. and R.W. Davis. 1983. Efficient isolation of genes by using antibody probes. Proc. Natl. Acad. Sci. 80: 1194 1198.

Zink, B. and R. Paro. 1989. In vivo binding pattern of a transregulator of homeotic genes in Drosophila melanogaster. Nature 337: 468-471. 


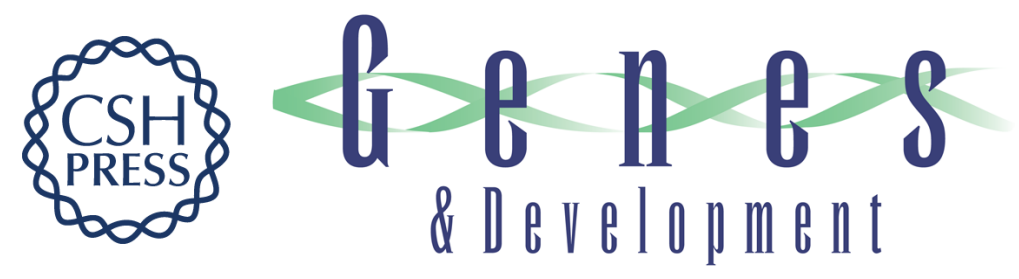

\section{Characterization of a Drosophila protein associated with boundaries of transcriptionally active chromatin.}

D T Champlin, M Frasch, H Saumweber, et al.

Genes Dev. 1991, 5:

Access the most recent version at doi:10.1101/gad.5.9.1611

References This article cites 51 articles, 18 of which can be accessed free at: http://genesdev.cshlp.org/content/5/9/1611.full.htmI\#ref-list-1

License

Email Alerting

Receive free email alerts when new articles cite this article - sign up in the box at the top Service right corner of the article or click here.

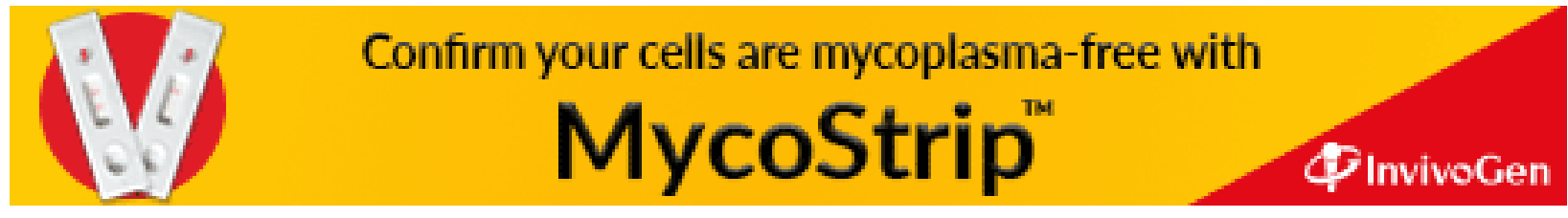

